\title{
¿Pueden las autoevaluaciones del alumnado ser la nota final de una asignatura?: el caso de biogerontologia
}

\author{
Dámaso Crespo \\ Departamento de Anatomía y Biología Celular, Facultad de Medicina. Universidad de Cantabria
}

Presentamos un estudio de la correlación entre las calificaciones objetivas $(\mathrm{CO})$ en una prueba tipo test de los estudiantes en la asignatura optativa Biogerontología con las autoevaluaciones subjetivas (AS) que los alumnos se asignaron tras la realización de la prueba y la que consideraron se merecían con relación al esfuerzo (AE) realizado para preparar dicha materia. El estadístico utilizado ha sido el coeficiente de correlación (rxy). Los resultados obtenidos muestran una alta correlación $\mathbf{r x y}=0,77$ cuando se comparan AS-AE. Sin embargo, rxy es muy bajo para los pares de valores CO-AS(0,05) y CO-AE (-0,07). Existen diferencias significativas $(\mathbf{p}<0,05)$ cuando se comparan los pares de valores CO-AS y CO-AE, mientras que no hay diferencia al comparar AS-AE. Los resultados de este estudio sugieren que las valoraciones AS y AE no pueden ser utilizadas como criterio final de calificación en este grupo de estudiant es por presentar ambas una correlación muy baja con respecto a la $\mathrm{CO}$ obtenida en la prueba de examen.

Palabras clave:

Au to evaluación. Meto dolo gía. Biog er on tolo gía. Asig na tu ra optativa
Is it possible to assume the autoevaluation of the students as the final mark of a subject?: the case of biogerontology.

This study correlates the marks obtained in the test of the optative subject Biogerontology (CO), and those self-attributed by a group of students upon level of study (AS) and dedication (AE) to the subject.

Statistical analysis used the correlation coefficient ( $r x y$ ). The results showed a low correlation when CO-AS (0.05) and CO-AE (-0.07) marks were compared (p<0.05). A high value (0.77) was obtained when both subjective marks were compared (AS-AE). Our results well suggest that subjective marks should not be used as the final marks in this subject.

Key words:

Self-evaluation. Methodology. Biogerontology. Optative Subject.

\section{INTRODUCCIÓN}

La implantación de un nuevo plan de estudios en las Facultades de Medicina ha supuesto, entre otras novedades, la introducción de un grupo heterogéneo de asignaturas optativas cuyo objetivo fundamental

\footnotetext{
$\overline{\text { Correspondencia: }}$

Dámaso Crespo

Departamento de Anatomía y Biología Celular

Facultad de Medicina. Universidad de Cantabria

Avenida del Cardenal Herrera Oria s/n

Teléfono 942201927

Fax 942201903

e-mail: crespod@unican.es
}

es acercar al alumnado que las cursa a conocimientos que no son objeto de estudio de las materias troncales y obligatorias. La asignatura optativa Biogerontología se imparte en el primer cuatrimestre del tercer curso de la Licenciatura en Medicina de la Universidad de Cantabria y tiene como objetivo fundamental introducir al alumnado en el conocimiento de las bases biomédicas del proceso de envejecimiento ${ }^{1}$. Durante los cuatro cursos que se ha venido impartiendo, el número medio de alumnos matriculados ha sido 66 y su evaluación se ha realizado por medio de una prueba objetiva tipo test en la cual había 20 preguntas con dos opciones posibles (verdadero o falso). La calificación final se 
obtiene aplicando la fórmula $A-(E / 2)$ y se requiere un mínimo de 10 puntos para superar la materia.

Respecto del sistema de evaluación de los conocimientos una consideración realizada, con frecuencia, por los estudiantes ha sido que la cal ificación de la asignatura debía hacerse atendiendo a otros factores entre los que señalan: asistencia a clase, trabajo práctico opcional, revisión bibliográfica, su propia valoración, etc. Bajo esta premisa nos planteamos en el cur so 2000-2001 eval uar la posibilidad deque una vez realizada la prueba objetiva ti po test, el alumnado pu diera autoevaluarse con respecto a la nota que esperaba obtener a la vista de la realización de su examen (AS) y también con respecto al esfuerzo (AE) realizado para superar la materia (asistencia a dase, horas de estudio, consultas bibliográficas, etc). El objetivo de este estudio era exami nar la posibilidad de que la calificación final de la asignatura Biogerontología pudiera obtenerse por medio de la autovaloración subjetiva del alumnado² en vez de someter a este a una prueba objetiva de valoración ${ }^{3,4,5}$. Para ello utilizamos el coeficiente de correlación $(r x y)^{6}$. Finalmente se contrasta, con un nivel de significación $\alpha=0,05$, la hi pótesis nula de que no existe una relación lineal entre los pares de variables CO-AS, CO-AE y AS-AE (H 0:rxy =0) frente a la alternativa de que dicha relación existe $(\mathrm{H}$ 1:rxy $\neq 0)$.

\section{ATERIAL Y METODOS}

El total de alumnos matriculados $(N=61)$ en el aurso 2000-2001 de la asignatura Biogerontología se presentaron al examen final de la misma. Los alumnos conoáan el tipo de prueba y la fórmula de calificación de los contenidos impartidos. En la hoja derespuestas se introdujer on dos preguntas a las cuales el alumno debía contestar, si lo consider aba oportuno, con una valoración entre el 0 y el 10. Si deseaba utilizar decimales se señalaba que estos debían ser 25 , 50 o 75. Las dos preguntas señaladas cor respondían a los siguientes enunciados. La primera, autoevaluacón subjetiva (AS) era: "A la vista del examen que usted acaba derealizar cre que su puntuadón será". La segunda pregunta, autoevaluación del esfuerzo $(\mathrm{AE})$, era: "Considero que dada mi asistencia a clase, consulta de bibliografía y esfuerzo general en la preparación de la materia, mi calificación debe ser". Un total de 36 alumnos $(n=36)$, que representan el $59 \%$ de los matriculados contestaron a las dos preguntas y sólo ellos fueron utilizados para este estudio (16 alumnos contestaron sólo a una de las dos preguntas y 9 no contestaron a ninguna).
Con los datos dbtenidos se procedió, en primer lugar, a efectuar un análisis de correlación entre los valores obtenidos en las tres calificaciones (CO, AS, y $A E$ ). Para ello se utilizó como estadístico el coeficientedecorrelación de Pearson (rxy) cuyo valor tiene un rango comprendido entre 1 y -1 . En valor 1 indica una correlación perfecta y positiva, por otra parte, el valor -1 indica una corr dación perfecta y negativa entre los dos grupos de val ores comparados. L os valores próximos a 0 se consideran carentes de toda correlación. Así, se procedió a la dbtención de los valores de rxy para los pares CO-AS, CO-AE y AS-AE.

Con el objetivo de determinar la existencia de posi bles diferencias signi ficati vas entre los diferentes valores de los coeficientes, estos se procesaron estadísticamente y se utilizó un nivel de significación $\alpha=0,05$ para contrastar la hipótesi s nul a (H0) de que no existe una relación lineal entre $x$ e y.

\section{RESULTADOS Y DISCUSIÓN}

El total de los alumnos presentados al examen $(\mathrm{N}=61)$ demostraron poseer los conocimientos exigidos para superar la materia. De estos, aquellos que entraron a formar parte de este estudio $(n=36)$ por haber contestado voluntariamente a las dos preguntas sobre sus autoval oraciones (AS y AE) obtuvieron una nota media en la calificación de la prueba objetiva (CO) de 7,34 $\pm 1,07$ (media \pm desviación típica), en $A S(7,45 \pm 1,11)$ y en $A E(7,9 \pm 1,18)$.

Los resultados de nuestros estudios para la asignatura Biogerontología destacan los bajos valores obtenidos al comparar los pares de variables CO-AS $(0,05)$ y CO-AE $(-0,07)$, mientras que el rxy del par CS-CE alcanzó un valor de 0,77.

El test de hipótesis con un nivel de significación de $\alpha=0,05$, sobre si existe o no una relación lineal, resultó significativo para los pares de variables $\mathrm{CO}$ AS y CO-AE $(p<0,05)$ y no significativo en el caso de AS-AE.

La progresiva implantación de los nuevos planes de estudio en las Facultades de Medicina ha supuesto, entre otras novedades, la división de las materias a i mpartir en tres grupos de asignaturas: troncales, obligatorias y optati vas, pu di endo estas últimas ser cursadas de forma vol untaria por el alumnado en el nuevo di seño curricular 7,8,9. Este aspecto introduce un cier to grado de motivad ón o apetencia por la adquisición de los conocimientos que en ellas se imparten?.

En la asignatura de Biogerontología una consi deración que realizan los alumnos es que no debía 
haber una prueba objetiva (test) para su calificación ya que este tipo de pruebas introducen altos componentes de estrés y ansi edad y que la evaluación se debía efectuar por otros métodos ${ }^{2}$. Con el objetivo de analizar la posibilidad de introducir una nueva forma de eval uación basada en oriterios subjetivos del alumnado, en el curso académico (2000-2001), hemos introducido en la prueba objetiva la posibilidad de que el al umno se evaluara subjetivamente tanto respecto a sus conocimi entos (CS) como a su esfuerzo en la materia (CE).

El análisis estadístico mostró la existencia de diferencias significativas $(p \varangle 0,05)$ y por lo tanto el rechazo de la hipótesis nula. Por otra parte no hubo diferencias significativas entre AS y $\operatorname{AE}(p<0,05)$. Este alto valor para el par AS-AE ( $r x y=0,77)$, no es de extrañar, pues refleja la valoración por parte del estudiante de dos vertientes de su propia subjetividad. En este sentido este al to val or nos indica que el estudiante se val ora de forma consistente respecto a dicha subjetividad.

Desde el punto de vista del docente uni versitario Ilama la atendi ón la baja corr elación entre la cal ificación objetiva (CO) que el alumnado obtiene en una prueba tipo test y las valoraciones que el se otorga (AS y AE). El análisis de estos datos muestra que los alumnos que obtuvieron mejores cal ificaciones objetivas expresaron subjeti vamente un menor esfuerzo para superar la asignatura y un peor resultado en la prueba, lo que denota por parte de este grupo de estudiantes un sesgo de infravaloración de su esfuerzo personal. Por el contrario, los al umnos con calificaciones objetivas más bajas se puntuaban valores subjetivos y de esfuerzo más altos, indicando una sobrevaloración de su parti dpación en el ren di mi ento de la asignatura.

Toda posible mejora en el proceso de eval uaci ón del alumnado debe ser analizado. En este sentido, la autoevaluación pudiera ser considerada como una opción válida, aunque su utilización está muy restringida al ámbi to preuniversi tario ${ }^{10}$. Consideramos que los resultados que hemos obtenido en este estudio no animan a cal ificar a los alumnos por medio de pr uebas subjetivas en las que ellos mismos seevalúen por existir importantes diferen cias entreel rendimiento medido de forma objetiva y el rendimiento que el alumnado se atribuye de forma subjeti va.

\section{AGRADECIMIENTO}

El autor quiere agradecer a $\mathrm{D}^{\mathfrak{a}}$ Carmen Chasco por su ayuda en la búsqueda bibliográfica y a los alumnos que han participado en este estudio su amabilidad en contestar a las cuestiones preguntadas y entender que el profesor no podía efectuar ningún comentario al respecto.

\section{BIBLIOGRAFÍA}

1. Resolución de 2 de agosto de 1994 de la U ni-versidad de Cantabria, por la que se ordena la publicación del plan de estudios de Licenciado en Medicina, a impartir en la Facultad de Medicina de esta Universidad. BOE 1994; 214:2800928021.

2. Fernández-Ballesteros R. Anatomía de los autoinformes. Evaluación Psicológica 1991; 3:263-290.

3. Palés J L. Análisis del proceso de implantación de los nuevos planes de estudio de la Licenciatura de Medicina. Educación Médica 1999; 2:102-104.

4. Polo A, Hernández J M, Pozo, C. Evaluación del estrés académico en estudiantes universitarios. Ansiedad y Estrés 1995; 2:159-172.

5. De la Cruz Tomé MA. Evaluación del conocimiento y su adquisición. Introducción a la Evaluación Psicológica I. Ed. Pirámide, 1999.

6. Martínez-Arias M, coordinador. Psicología Matemática II. Vol. 2. UNED, Madrid, 1990.

7. Valle A, González R, Núñez J C, Vieiro P. Un modelo cognitivo-motivacional explicativo del rendimiento académico en la universidad. Estudios de Psicología 1999; 62:77-100.

8. Materson D. El aprendizaje en las Facultades de Medicina: una perspectiva sobre la situación actual. Educación Médica 1998; 1:7-12.

9. Graell S, Pérez J . Asistencia a clase y rendimiento académico. XIV Congreso Nacional de la Sociedad Española de Educación Médica. Educación Médica 1999; 2:150.

10. Castro J A, García Prada J M, Arias MA, Barrientos $B, P e ́ r e z ~ M L$. La valoración que los adolescentes hacen de su rendimiento académico. Revista de Ciencias de la Educación. 1997; 171:311-333. 